\title{
Multicriterial Analysis of Explosives in the THz Range
}

\begin{abstract}
R. RynieC*, M. Piszczek and M. Szustakowski
Institute of Optoelectronics, Military University of Technology, S. Kaliskiego 2, 00-908 Warsaw, Poland

The increasing interest in the development of identification methods of explosives in $\mathrm{THz}$ region over the past few years has been observed because of potential terrorist threats. To determine specific spectra properties of materials both possible spectroscopic methods - time domain spectroscopy and Fourier transform infrared spectroscopy are used to analyze different classes of compounds. This paper deals with an identification problem of more than one compound. This task was solved mathematically by means of the following methods: Monte Carlo including principal components analysis methods and analytic methods. Investigations were limited to a detector, which based on a single-frequency quantum dot matrix in $0.3 \div 1.3 \mathrm{THz}$ range. The mathematical analysis also includes the atmosphere influence on propagation of $\mathrm{THz}$ waves. By means of the presented methods we indicated fourteen optimal frequency points and next we managed to identify one explosive from a set of ten explosives.
\end{abstract}

PACS numbers: 42.81.Bm, 42.81.Cn, 42.81.Dp

\section{Introduction}

THz waves lie between microwave and infrared [1]. Typically $\mathrm{THz}$ band refers to the spectrum region between $300 \mathrm{GHz}$ to $10 \mathrm{THz}$. There are unique properties of $\mathrm{THz}$ radiation that makes it potentially powerful technique in security screening [2-5]. The most important, $\mathrm{THz}$ radiation penetrates many non-conducting materials. Additionally, this radiation is characterized by low energy and it is non-ionizing and no-invasive to biological tissue. Finally, many chemical substances exhibit characteristic spectral response at $\mathrm{THz}$ frequencies that can be used to identify threat materials even when hidden in packages or under clothes by means of spectroscopy $[6,7]$. Terahertz technology is gradually maturing and already used in many applications, particularly within science. At planning stage is the application of new technologies in short-range high-resolution radar, military applications and surveillance systems, notably security systems. Unfortunately, the atmosphere influence limits propagation to few meters. There are, however, the narrow windows of good transmission. In this paper procedures used to determine optimum frequency locations of limited numbers of data points for identification of ten different explosives are presented.

\section{Measurements}

Samples (pellets) of explosives were prepared by mixing $360 \mathrm{mg}$ of teflon and $40 \mathrm{mg}(10 \%)$ of the explosive and compressing the mixture into pellets with $13 \mathrm{~mm}$ diameter [7]. All measurements were performed in transmission mode with a FTIR spectrometer Brucker 113v with $1 \mathrm{~cm}^{-1}$ resolution (Fig. 1). We measured absorbance $(A)$

\footnotetext{
* corresponding author; e-mail: rryniec@wat.edu.pl
}

defined as:

$$
A=-\log \left(I / I_{0}\right)
$$

where $I$ - transmitted light intensity, $I_{0}$ - the intensity of the light before it enters the sample.

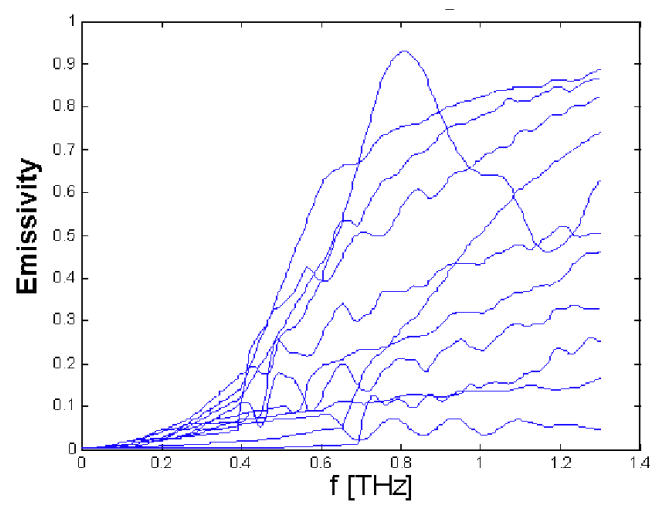

Fig. 1. Collection of ten explosives (AN, ammonium nitrate, HMDT, HMX, PETN, RDX, saletrol, TATP, TNT, explosives emulsion).

Neglecting the influence of reflection and refraction we can calculate emissivity $(\varepsilon)$ as

$$
\varepsilon=1-\frac{I}{I_{0}}=1-10^{(-A)}
$$

In the terahertz region many strong absorption lines of water vapor are observed. Figure 2 presents calculation of transmission windows made with use of Hitran database, with resolutions $0.1 \mathrm{~cm}^{-1}(3 \mathrm{GHz})$, for the reference atmosphere with pressure of $1 \mathrm{~atm}$, temperature of $293.15 \mathrm{~K}$ and water vapor density of $10 \mathrm{~g} / \mathrm{m}^{3}$, which corresponds to humidity of about $54 \%$. The propagation path is 2 meter long. Typical humidity inside a room is about $48 \div 55 \%$. 


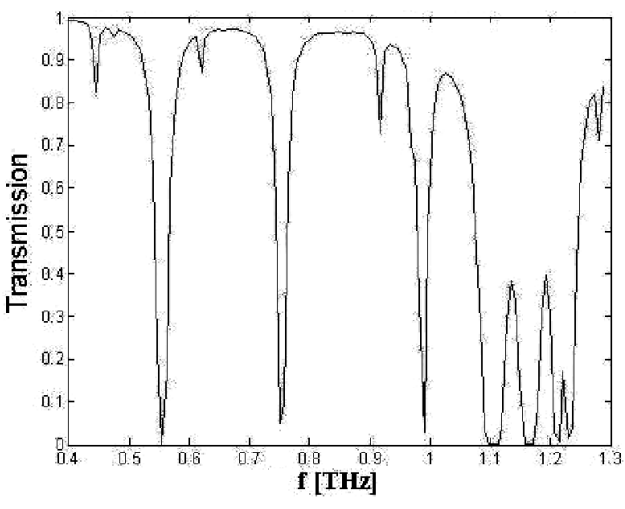

Fig. 2. Attenuation of the atmosphere in the range $0.4 \div 1.3 \mathrm{THz}$ (293 K, 50\% humidity).

\section{Goal}

Knowledge of the signal reaching the detector is important from the point of view of a designer of the detection and identification systems. Attenuation of the atmosphere significantly influences the spectrum of $\mathrm{THz}$ wave propagating in air which is presented in Fig. 3. In order to reduce the influence of atmospheric attenuation, during the identification process, to a minimum, it is necessary to indicate the frequencies with good atmospheric transmission.

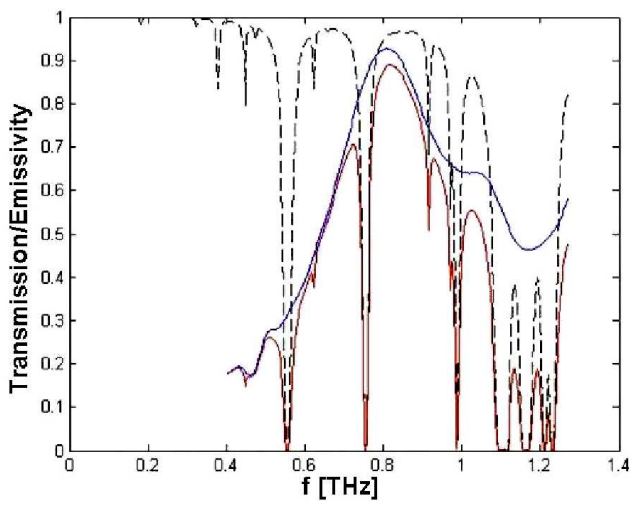

Fig. 3. RDX spectrum measured in vacuum (blue), distorted by atmosphere attenuation (red). Dotted line represents transmission of the atmosphere.

Unfortunately, uniform distribution of the limited number of measurement points can lose information due to the atmosphere attenuation. Statistical method based on the Monte Carlo was applied to solve the problem of determination the optimum location of narrow-band (about $10 \mathrm{GHz}$ ) detectors. All of explosive spectra were transformed before. The procedure for preparation of spectra for the numerical analysis was carried out using the following methodology:

1. spectra of all explosives $X$ were interpolated at the points calculated by the Hitran Base for atmospheric attenuation,
2. then convoluted with the spectrum of the atmospheric attenuation obtained spectra were normalized by min-max normalization described by formula (3):

$$
X^{\prime}=\frac{X-\min (X)}{\max (X)-\min (X)} .
$$

The signals prepared in this way were further analyzed.

\section{Numerical analysis}

In our study we used a principal components analysis (PCA) method. PCA is a useful statistical technique that was applied in face recognition. It bases on identification patterns in data and expresses the data in such a way as to highlight their similarities and differences. The main advantage of PCA is possible to compress the data by reduction the number of dimensions without much loss of set of data. The procedure of finding the optimal distribution of frequencies of measure points was conducted using the following methodology:

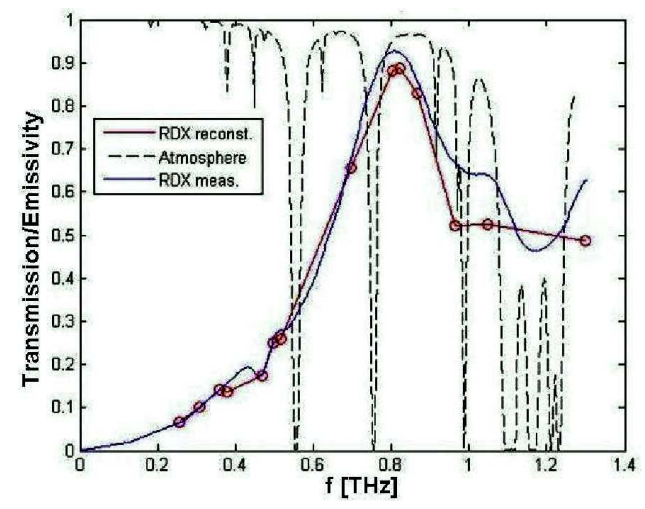

Fig. 4. Reconstruction of RDX spectrum by limited number of points.

1. collections of $5 \mathrm{k}, 10 \mathrm{k}$ and $20 \mathrm{k}$ sets of 14 elements of random variables was determined by the Matlab generator of random numbers. Each element of the set represents the mean frequency of the definite detector;

2. calculation of the sum of absolute differences (SAD):

$$
\mathrm{SAD}=\sum_{i=1}^{n}\left|X_{i}-X_{i}^{\prime}\right|,
$$

and the sum of squared differences (SSD) (5):

$$
\mathrm{SSD}=\sum_{i=1}^{n}\left(X_{i}-X_{i}^{\prime}\right)^{2},
$$

where $X_{i}$ - reference signal (measured in vacuum), $X_{i}^{\prime}$ - distorted signal by the atmosphere attenuation, $n=368$; computation was carried out for interpolated signals for each set of 14 frequencies;

3. comparison of results revealed that it is sufficient to analyze 10k sets of 14 frequencies; 
4. creation the $10 \mathrm{k} \times 10$ matrix was carried out. Elements of the matrix were SSD and SAD computed for every set of 14 frequencies;

5 . elements of matrix were standardized by formula

$$
X^{*}=\frac{X-\bar{X}}{\sigma(X)},
$$

where $\sigma$ - standard deviation, $X-\mathrm{SAD}$ or SSD, $\bar{X}-\operatorname{mean} X$;

6. calculation the covariance matrix $10 \times 10$ by formula

$$
\begin{gathered}
C=\left[\begin{array}{cccc}
\operatorname{cov}\left(x_{1} x_{1}\right) & \operatorname{cov}\left(x_{1} x_{2}\right) & \ldots & \operatorname{cov}\left(x_{1} x_{n}\right) \\
\operatorname{cov}\left(x_{2} x_{1}\right) & \operatorname{cov}\left(x_{2} x_{2}\right) & \ldots & \operatorname{cov}\left(x_{2} x_{n}\right) \\
\vdots & \vdots & \vdots & \vdots \\
\operatorname{cov}\left(x_{n} x_{1}\right) & \operatorname{cov}\left(x_{n} x_{2}\right) & \ldots & \operatorname{cov}\left(x_{n} x_{n}\right)
\end{array}\right] \\
\operatorname{cov}(x y)=\frac{\sum_{i=1}^{N}\left(X_{i}-X^{*}\right)\left(Y_{i}-Y^{*}\right)}{(n-1)},
\end{gathered}
$$

where $X, Y$ - standardized SAD or SSD values, $n=10$ (every explosive); $N$ - number of measure points;

7. calculation the eigenvectors and eigenvalues of the covariance matrix.

Estimation minimizing the value of eigenvalues of covariance matrix allowed to obtain fourteen frequency points which are possible to reconstruct all of ten compounds with a high quality. Identification process is based on comparison of the characteristic properties of the measured signal and the reference. The challenge is the definition of characteristic properties of compounds which allow discrimination of them. PCA method was used to solve this problem just as previous. The covariance matrix for 10k sets of pair of compounds was calculated by formula (9):

$$
P=\sum_{i=1}^{n}\left(X_{i}-Y_{i}\right)^{2},
$$

where $n=$ number of measure points; $X, Y$ - interpolated spectra for every set of 14 frequencies.

The optimum location of detectors for separation of 10 compounds was estimated by minimizing the inversion of sum $P$ value. The set of 14 frequencies was determined which allow both good quality reconstruction spectra and discrimination, then. Figure 4 shows 14 data measure points.

\section{Results and conclusions}

The identification process based on the comparison method is limited by the background interference. In this study, authors proposed to use the SAD and SSD criterion to estimate the quality of reconstruction and the separation spectra of 10 explosives.

Results of identifications of the explosives.

TABLE

\begin{tabular}{c|c|c|c|c|c|c|c|c|c|c}
\hline \hline & RDX & HMX & TNT & HMDT & PETN & Emulsion & AN+AL & AN & TATP & ANFO \\
\hline RDX & $\mathbf{0 . 2 6 5 7}$ & 1.4783 & 1.9348 & 1.8753 & 1.8753 & 1.0867 & 0.7999 & 0.7930 & 2.1250 & 0.7637 \\
HMX & 1.6199 & $\mathbf{0 . 2 3 5 6}$ & 0.7498 & 0.8454 & 0.3753 & 0.5493 & 1.0999 & 1.6613 & 1.0924 & 1.3315 \\
TNT & 2.168 & 1.0393 & $\mathbf{0 . 0 7 8 5}$ & 0.2096 & 0.6802 & 1.0374 & 1.7807 & 2.3174 & 0.3072 & 2.0272 \\
HMDT & 2.1212 & 1.1220 & 0.2651 & $\mathbf{0 . 0 5 7 1}$ & 0.6761 & 1.0046 & 1.7593 & 2.2816 & 0.2292 & 2.0102 \\
PETN & 1.6554 & 0.6821 & 0.5188 & 0.4870 & $\mathbf{0 . 1 5 1 3}$ & 0.4924 & 1.2338 & 1.7654 & 0.7593 & 1.4843 \\
Emulsion & 1.3406 & 0.6924 & 0.8578 & 0.8016 & 0.3094 & $\mathbf{0 . 1 8 4 4}$ & 0.9340 & 1.4430 & 1.0682 & 1.1789 \\
AN+AL & 0.8205 & 0.8969 & 1.4992 & 1.4647 & 0.8903 & 0.5379 & $\mathbf{0 . 2 9 0 1}$ & 0.7980 & 1.7348 & 0.5431 \\
AN & 0.4921 & 1.3909 & 2.0067 & 1.9586 & 1.4024 & 1.0388 & 0.4655 & $\mathbf{0 . 3 3 7 2}$ & 2.2233 & 0.3636 \\
TATP & 2.3522 & 1.3310 & 0.3934 & 0.2942 & 0.9114 & 1.2417 & 1.9983 & 2.5193 & $\mathbf{0 . 0 2 0 2}$ & 2.2497 \\
ANFO & 0.6218 & 1.0666 & 1.7194 & 1.6916 & 1.1151 & 0.7595 & 0.2548 & 0.5678 & 1.9615 & $\mathbf{0 . 3 2 0 4}$ \\
& & & & & & & & &
\end{tabular}

This allowed determining a set of frequencies with the limited atmosphere attenuation during the identification process. The set of measurement points was obtained using statistical methods. To verify results of analysis, the reference spectra were deformed by the atmosphere transmission and then analyzed to identify by the Euclidean distance criteria (10) for 14 frequencies

$$
d_{\mathrm{E}}(x, y)=\sqrt{\sum_{i=1}^{14}\left(x_{i}-y_{i}\right)^{2}},
$$

where $x$ - deformed explosive spectrum, $y$ - reference explosive spectrum.

The application of this criterion made it possible to identify 10 explosives. The results are shown in Table.

As you can see, majority of materials were identified correctly. Thus, it is possible to distinguish explosives 
using 14 frequencies measure points by analysis of values of the signal reaching detector. In future the work algorithm for identification will be improved.

\section{Acknowledgments}

The analysis was carried out for The European Union project TERAEYE for specific number and type of the detectors.

\section{References}

[1] H.-B. Liu, Y. Chen, G.J. Bastiaans, X.-C. Zhang, Opt. Exp. 14, 415 (2006).
[2] K. Jasek, T. Budzynski, E. Stryszak, Acta Phys. Pol. A 116, 321 (2009).

[3] N. Palka, W. Ciurapinski, J. Wójcik, M. Szustakowski, Acta Phys. Pol. A 114, A-155 (2009).

[4] N. Pałka, M. Szustakowski, R. Ryniec, Elektronika 1, 3 (2010).

[5] A. Rogalski, Acta Phys. Pol. A 116, 389 (2009).

[6] Z. Piotrowski, Acta Phys. Pol. A 116, 375 (2009).

[7] A. Giles Davies, Materials Today 11, 11 (2008). 